\title{
ANÁLISE DE IMAGENS NA DETERMINAÇÃO DA FORMA E TEXTURA DE AREIAS
}

\author{
Georgia Serafim Araújo(1), Kátia Vanessa Bicalho(2)* e Fernando Avancini \\ Tristão(2)
}

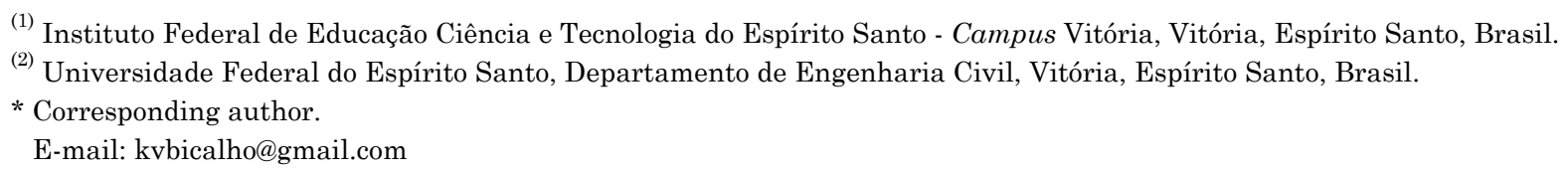

\section{RESUMO}

A forma dos grãos tem importante papel na avaliação da distribuição granulométrica das partículas de um solo. Entretanto, a maioria das técnicas de peneiramento evidencia que os grãos são esféricos e descritos por um único parâmetro, o diâmetro. Este trabalho teve por objetivo estudar a aplicação da análise de imagens, aliada ao grau de esfericidade e à teoria dos fractais, como ferramenta para mensurar parâmetros de forma e textura superficial de duas areias de características supostamente distintas da região da Grande Vitória, Espírito Santo, Brasil. Os resultados dos parâmetros de forma e textura superficial das amostras são apresentados e discutidos. As análises dos resultados para a variável esfericidade mostram diferenças estatísticas significativas entre as duas areias de origens diferentes. A areia de jazida de cordão litorâneo (AJ) apresentou grau de esfericidade ligeiramente superior e textura superficial mais lisa do que a de rio (AR), indicando que as areias AJ sofreram maior intemperismo em razão do transporte.

Palavras-chave: parâmetros texturais, análise computacional, teoria dos fractais.

\section{ABSTRACT: USE OF IMAGE ANALYSIS TO DETERMINE THE SHAPE AND TEXTURE OF SANDS}

The Shape of sand grains plays an important role in evaluation of soil particle size distribution. However, in most sieving techniques it is assumed that grains are spherical and can be described by a single parameter, diameter. Moreover, the subjectivity of the tests used to estimate sand roughness 


\begin{abstract}
makes it difficult to obtain data that accurately represent reality. The aim of this paper was to study the use of image analysis together with the degree of sphericity and fractal theory as a tool to measure the textural parameters of the shape and surface texture of two types of quartz sands with supposedly distinct characteristics from the region of Vitória, Espírito Santo, Brazil. The results of the shape and surface texture parameters of the samples are presented and discussed. Statistical analyses of the results for the sphericity variable showed significant statistical differences between the two sands of different origins. The results show that river sands $(R S)$, though having been subjected to a greater degree of transport and attrition, do not have a greater degree of roundness and a smoother surface texture than sands from the coastal spit (BS). The values obtained in the test were statistically analyzed, and again it was confirmed that the sand of the BS has a slightly greater sphericity and smoother surface texture than that of the RS, indicating that the BS sands underwent greater weathering due to transport.
\end{abstract}

Keywords: textural parameters, computational analysis, fractal theory.

\section{INTRODUÇÃO}

A determinação da distribuição do tamanho das partículas (análise granulométrica) e dos parâmetros de forma e textura superficial de um solo é variável estudada em áreas como física do solo, geologia e engenharia civil. Essa variável tem aplicações práticas nos estudos de drenagem, erosão e capacidade de retenção de água de um solo, podendo, inclusive, indicar a origem e o desgaste sofrido no transporte por esse solo.

A análise da influência dos aspectos geométricos dos grãos de solo normalmente se restringe à composição granulométrica avaliada pelo ensaio de peneiramento. Entretanto, esse ensaio não permite obter outros parâmetros como a forma e rugosidade, por exemplo. Kwan et al. (1999) e Maerz (2004) alertaram que, na interpretação dos resultados de granulometria obtida mediante o peneiramento, é preciso considerar que partículas que passam pela peneira de malha quadrada podem ter dimensões maiores do que a abertura de malha.

Geralmente, o procedimento para avaliação da morfologia dos grãos de areia é feito por comparação visual com uma carta-padrão em que se verifica o grau de arredondamento e a textura superficial grão a grão (Mclane, 1995). Entretanto, além de demorada, essa técnica de ensaio acaba por ser subjetiva e muito dependente da experiência do operador.

Como alternativa para minimizar as deficiências do ensaio de peneiramento, no que se refere à forma dos grãos, pode-se utilizar o grau de esfericidade, que é o índice de forma mais comum e que dá a ideia de quão próximo o grão está de uma esfera. A esfericidade possui diversas definições, entre elas, a apresentada por Mendes (1972) que definiu a esfericidade como a relação entre a área superficial da partícula e o seu volume; numericamente, essa medida indica o quão próximo o grão está de uma esfera em que os valores de $\mathrm{x}$, y e $\mathrm{z}$ nos eixos ortogonais são iguais. Para Mora e Kwan (2000), a esfericidade é a razão da área superficial de uma esfera de mesmo volume da partícula e a sua área real. Já Rittenhouse (1943) definiu esfericidade como a relação entre o diâmetro do círculo com uma área igual à projeção da partícula e ao diâmetro do menor círculo circunscrito à partícula, podendo variar de 0 a 1 ; na prática esses valores vão de 0,45 (alongada) a 0,97 (muito esférica).

A textura superficial de um grão de solo pode variar desde muito angular (superfície rugosa) até muito arredondado (superfície lisa) em razão das características do agente transportador. A verificação da textura é feita por comparação com uma carta-padrão, método demorado, subjetivo e difícil de automatizar.

As exigências de prazos reduzidos e a maior precisão nas medidas estimulam o uso de técnicas de medições alternativas ao peneiramento e ao método comparativo como a análise de imagens (Brzezicki e Kasperkiewicz, 1999; Lundqvist e Akersson, 2001). Essa técnica vem sendo especialmente impulsionada pelos avanços tecnológicos nos campos da informática e da eletrônica e no desenvolvimento de computadores pessoais cada vez mais potentes e acessíveis. A junção de todos esses fatores permite capturar, manipular e armazenar imagens com resoluções cada vez maiores e, inclusive, análises tridimensionais (Fernlund, 2005).

Uma alternativa é o emprego da teoria dos fractais para avaliar esse parâmetro. Fractal é um termo usado na geometria para descrever a forma de um objeto que não possui uma topologia ideal. A geometria fractal é uma ferramenta matemática para lidar com sistemas complexos que não têm características próprias a escalas diferentes (Carr et al., 1990; Bunde e Havlin, 1995). Hyslip e Vallejo (1997) apresentaram um método denominado área-perímetro para a avaliação da rugosidade de materiais granulares a partir da dimensão fractal de rugosidade $\left(D_{R}\right)$, obtida pela equação 1 :

$$
D_{R}=2 / m
$$


em que $m$ é o coeficiente angular ou inclinação da reta (log área/log perímetro). Os resultados indicaram tendência de areias mais rugosas apresentarem $\mathrm{D}_{\mathrm{R}}$ maiores. Scarlett (1996) também concordou que maiores valores de $\mathrm{D}_{\mathrm{R}}$ implicam em amostras mais rugosas.

Desse modo, a pesquisa teve por objetivos avaliar a eficácia da análise de imagens bidimensional e da teoria dos fractais como ferramentas na obtenção das medidas requeridas no cálculo da esfericidade e da rugosidade de solos e comparar os aspectos texturais de duas areias do Estado do Espírito Santo: uma de rio $(\mathrm{AR})$ e outra procedente de jazida de cordão litorâneo (AJ).

\section{MATERIAL E MÉTODOS}

Para avaliar a forma e rugosidade dos grãos de areia, foram selecionadas uma amostra de areia de rio (AR) e outra procedente de jazida próxima à praia (AJ). Supostamente, essas possuem características distintas em razão dos diferentes agentes transportadores e ambientes de sedimentação. A amostra AR foi extraída do rio Doce, no município de Colatina, ES, sendo a coleta feita em Vitória, ES, para onde a areia é transportada por via férrea para ser comercializada. A amostra AJ é proveniente de jazida próxima à praia e foi coletada na região da Barra do Jucu, no município de Vila Velha, ES. As análises foram realizadas no Laboratório de Materiais da Universidade Federal do Espírito Santo.

De acordo com o Sistema Unificado de Classificação dos Solos (SUC), as duas areias foram classificadas como SP, areias mal-graduadas. Com base no ensaio de peneiramento, o coeficiente de uniformidade, $\mathrm{Cu}$, que á a relação entre $\mathrm{D}_{60}$ (diâmetro correspondente

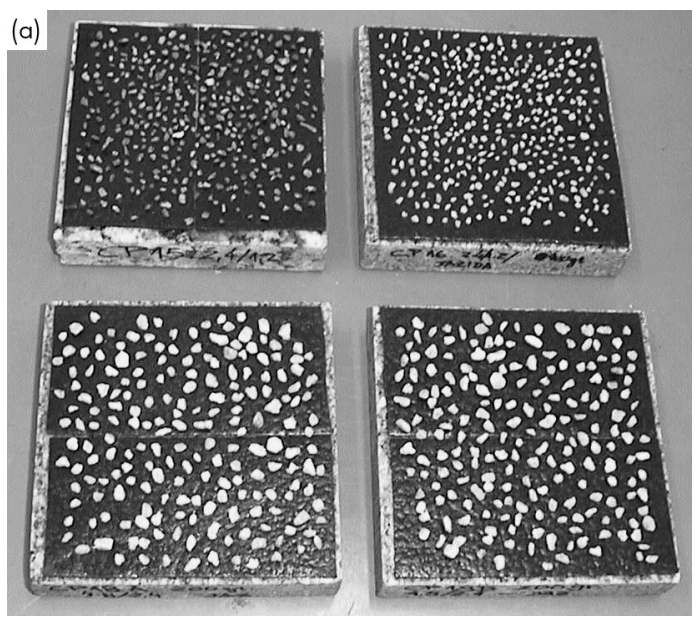

a $60 \%$ em peso total das partículas menores do que ele) e $\mathrm{D}_{10}$ (diâmetro correspondente a $10 \%$ em peso total das partículas menores do que ele) é maior para a areia de jazida (em torno de 6) do que para a de rio (cerca de 3). De acordo com a classificação SUC, areias uniformes têm $\mathrm{Cu}<6$. O mineral predominante nas duas areias ensaiadas é o quartzo; e densidade dos grãos, em torno de $2,65 \mathrm{~kg} \mathrm{dm}^{-3}$.

As areias ensaiadas foram separadas, pelo peneiramento, em seis frações conforme recomendação da NBR 248 (ABNT, 2003), a saber: 4,8-2,4; 2,4-1,2; $1,2-0,6 ; 0,6-0,3 ; 0,3-0,15 ;$ e 0,15-0,075 mm. Apesar das subdivisões das frações de areia consideradas neste trabalho serem diferentes das adotadas pela Embrapa (2012), as curvas granulométricas seguem tendência similar. Ressalta-se que neste trabalho todos grãos ficaram retidos na peneira $\mathrm{n}^{\circ} 200$ (abertura de malha $0,075 \mathrm{~mm}$ ), e são maiores que $0,05 \mathrm{~mm}$, não contendo, portanto, nenhum silte ou argila, mesmo pelo critério da Embrapa.

Para cada fração das duas areias, foi preparada uma amostra com determinada quantidade de grãos que fosse estatisticamente representativa. Com o objetivo de garantir a aleatoriedade na obtenção da imagem dos grãos, esses foram lançados em uma base adesiva fixada em uma superfície plana por meio de um funil. As amostras das frações mais grossas e mais finas são mostradas na figura 1a, 1b, respectivamente.

A obtenção dos parâmetros requeridos para verificar a forma dos grãos seguiu as seguintes etapas:

a. Fez-se aquisição das imagens de duas maneiras distintas: as três maiores frações foram fotografadas em estúdio com uma câmara fotográfica, e as imagens das frações menores foram obtidas com uma câmara digital acoplada a uma lupa (Figura 2);

b. Fotografou-se uma régua junto com os grãos para obter a escala das fotos (Figura 3);

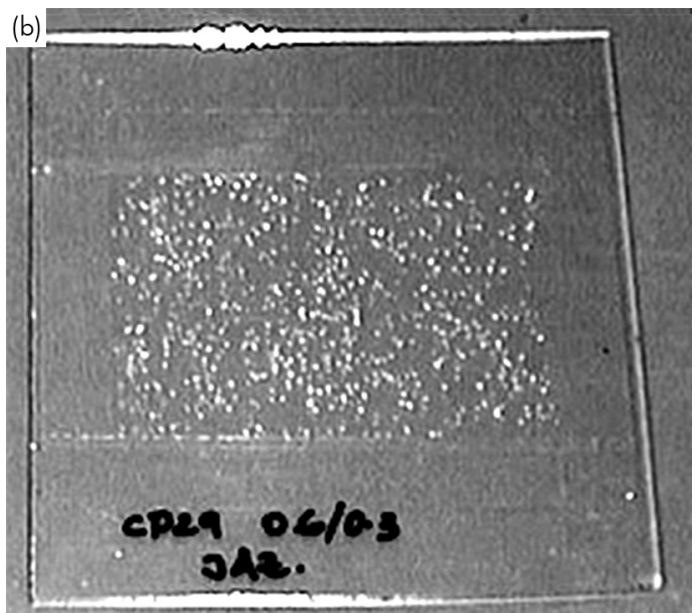

Figura 1. Amostras das frações de areia mais grossas (a) e mais finas (b) preparadas para aquisição das imagens. 
c. Analisaram-se, para cada fração, aproximadamente, 400 grãos, com base nos estudos de Persson (1998), o qual observou que amostras menores não permitiam análise estatística consistente;

d. Trataram-se as imagens ajustando o brilho e contraste para facilitar o reconhecimento das bordas dos grãos;

e. Detectaram-se as feições de interesse, no caso a borda dos grãos, e, ao final dessa operação, obteve-se uma imagem binária, que permite separar os contornos dos grãos do fundo; e

f. Fizeram-se as medições dos parâmetros requeridos para cálculo da esfericidade (áreas, perímetros e diâmetros equivalentes de uma circunferência), o tratamento estatístico (análise de variância) e a análise.

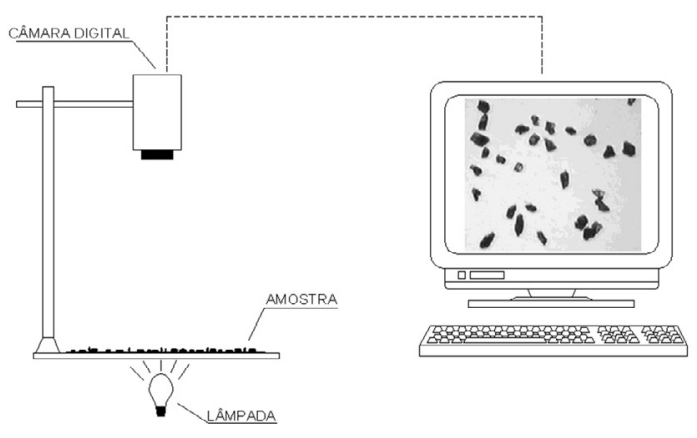

Figura 2. Esquema do aparato montado para fazer a aquisição das imagens das frações de areia mais finas.

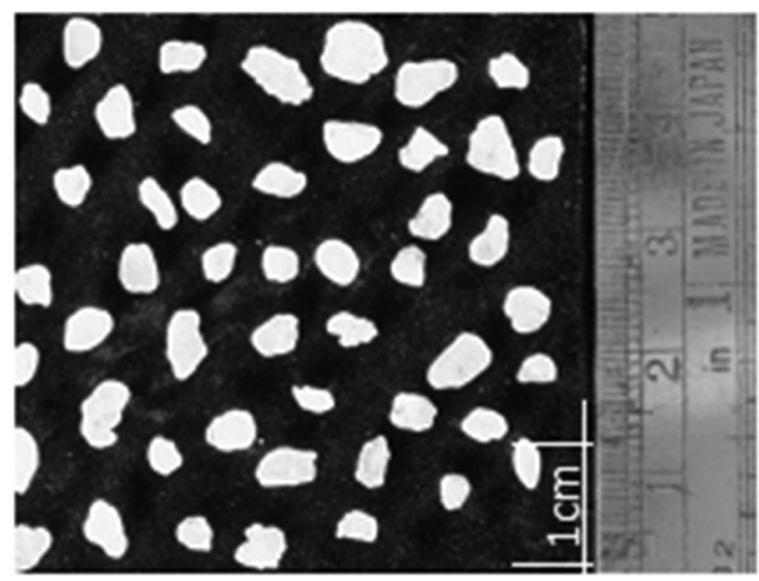

Figura 3. Amostra de areia das frações grossas com a escala auxiliar para referência do tamanho da imagem.

\section{RESULTADOS E DISCUSSÃO}

Para a avaliação dos graus de esfericidade das areias, empregou-se a definição apresentada por Rittenhouse (1943), visto que os parâmetros requeridos para o seu cálculo (diâmetro do círculo com uma área igual à projeção da partícula e o diâmetro do menor círculo circunscrito à partícula) podem ser obtidos de imagens bidimensionais.

A partir dos valores dos diâmetros da menor circunferência circunscrita e dos diâmetros equivalentes à área das projeções das partículas, obtidos na análise de imagens (Quadro 1), calculou-se a esfericidade média de cada fração que estão representadas graficamente na figura 4 .

Quadro 1. Valores médios dos diâmetros da menor circunferência circunscrita (DMCC) e dos diâmetros equivalentes de área (DEA) às projeções das partículas, para cálculo da esfericidade média de cada fração, de acordo com a definição de Ritenhouse (1943) areia do cordão litorâneo (AJ) e areia de rio (AR) da região de Vitória, ES

\begin{tabular}{lcccc}
\hline \multirow{2}{*}{ Fração } & \multicolumn{2}{c}{ DMCC } & \multicolumn{2}{c}{ DEA } \\
\cline { 2 - 5 } & AJ & AR & AJ & AR \\
\hline $\mathrm{mm}$ & \multicolumn{3}{c}{$\mathrm{mm}$} \\
$4,80-2,40$ & 4,56 & 4,93 & 3,696 & 3,869 \\
$2,40-1,20$ & 2,91 & 3,00 & 2,349 & 2,388 \\
$1,20-0,60$ & 1,78 & 1,47 & 1,381 & 1,162 \\
$0,60-0,30$ & 0,69 & 0,79 & 0,532 & 0,610 \\
$0,30-0,15$ & 0,37 & 0,44 & 0,295 & 0,343 \\
$0,15-0,075$ & 0,20 & 0,21 & 0,155 & 0,162 \\
\hline
\end{tabular}

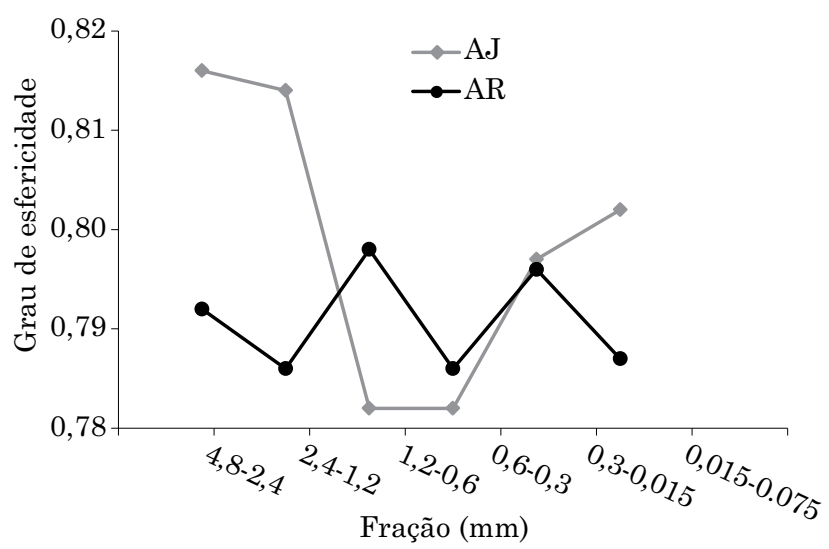

Figura 4. Valores médios dos graus de esfericidade, de acordo com a definição de Ritenhouse (1943), das frações da areia do cordão litorâneo (AJ) e areia de rio (AR) da região de Vitória, ES, obtidos pela análise de imagens. 


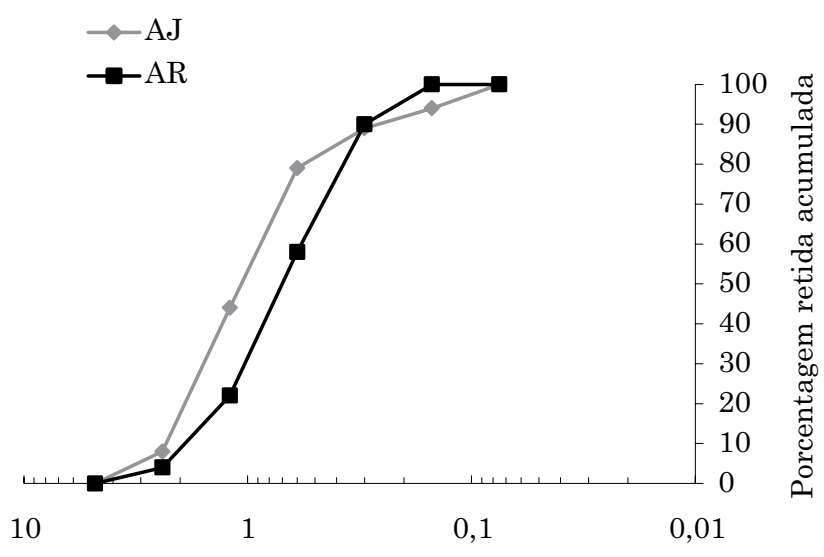

Abertura de malha (mm)

Figura 5. Curvas granulométricas empregadas para calcular a esfericidade média da areia do cordão litorâneo (AJ) e da areia de rio (AR).

(a)

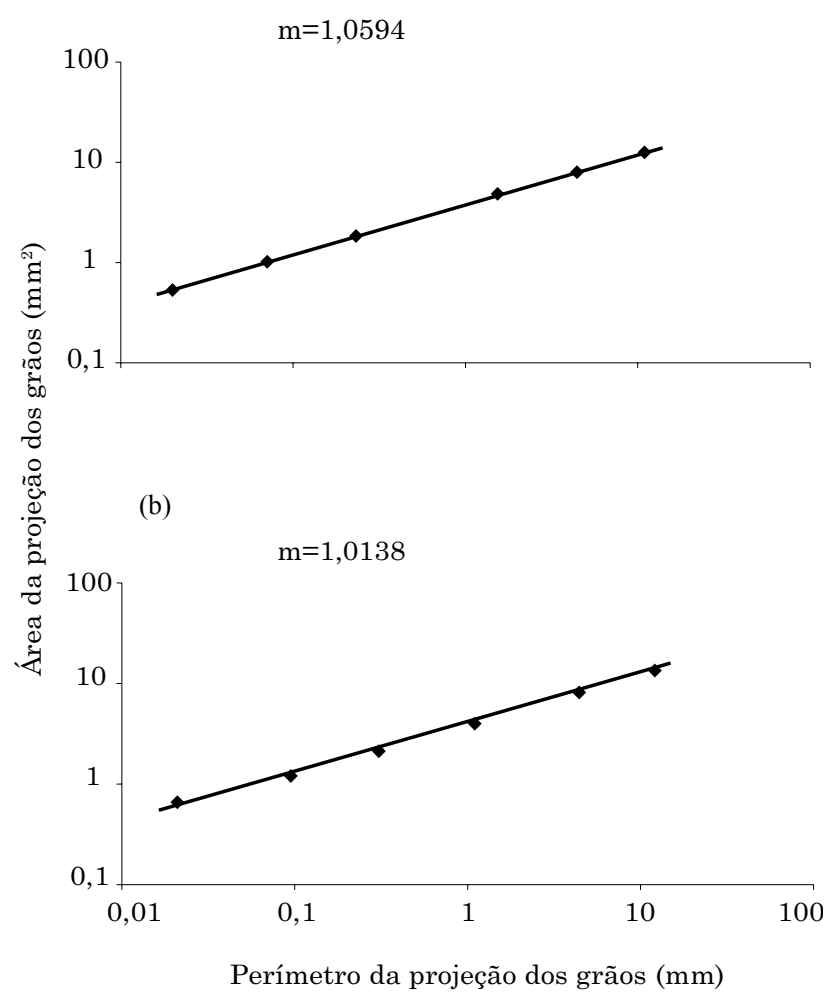

Figura 6. Relação entre a área e o perímetro de projeção dos grãos (escala logarítmica) cujo coeficiente angular (m) foi empregado para cálculo da dimensão fractal de rugosidade (DR). (a) Areia do cordão litorâneo (AJ), e (b) Areia de rio (AR) da região de Vitória, ES.
A areia AR evidenciou pequena variação na esfericidade, com tendência a apresentar valor próximo a 0,79 nas diversas frações estudadas. Já na areia AJ, verificaram-se três grupos de esfericidade bem distintos. As duas maiores frações (4,8-2,4 e 2,4-1,2 mm) possuíam grãos com a maior esfericidade, em torno de 0,815 . Nas duas frações seguintes (1,2-0,6 e 0,6-0,3 mm), os valores decresceram subitamente para $0,782 \mathrm{e}$ novamente aumentaram para um valor próximo de 0,800 nas duas frações mais finas $(0,3-0,15$ e 0,15-0,075 mm).

Se na prática a esfericidade varia de 0,45 (alongada) a 0,97 (muito esférica), é correto afirmar que as duas areias analisadas podem ser classificadas como esféricas.

Ao se calcular a esfericidade média das areias ensaiadas, aplicando-se as esfericidades médias das frações às porcentagens do material retido (Figura 5) em cada peneira, obteve-se que a areia de cordão litorâneo (AJ) apresentou valor de 0,792 ; e a de rio (AR), 0,786. Os valores de esfericidades médias são próximos dos valores obtidos no trabalho de Vepraskas e Cassel (1987) para diferentes areias.

Os resultados de esfericidade apresentados nos ensaios de análise de imagens confirmaram os resultados da análise morfoscópica realizada em 25 grãos de cada fração, escolhidos aleatoriamente por meio de uma lupa e classificando-os, por comparação, a partir de uma imagem-padrão de desgaste superficial e forma apresentados por Araújo (2001).

Os valores obtidos no ensaio foram analisados estatisticamente para a variável esfericidade com os fatores fração granulométrica e origem apresentando diferenças estatísticas significativas. As médias e os desvios-padrão das areias AR e AJ confirmaram que a AJ possui grau de esfericidade ligeiramente superior à $\mathrm{AR}$, indicando que as areias de jazida (AJ) sofreram maior intemperismo em razão do transporte.

Referente à textura superficial dos grãos dos solos arenosos, inicialmente se fez uma análise morfoscópica desses em que foi possível observar que na AJ $58 \%$ dos grãos são considerados lisos enquanto na AR esse percentual caiu para apenas $7 \%$.

Para verificar a correlação entre dimensão fractal de rugosidade, $\mathrm{D}_{\mathrm{R}}$, e rugosidade observada por Hyslip e Vallejo (1997), foram plotadas a figura 6 , em que foi possível calcular os valores de $D_{R}$ (dimensão fractal de rugosidade) em função do coeficiente angular da reta ajustada aos pontos, conforme equação 1 .

Para a AJ, a dimensão fractal de rugosidade calculada pelo coeficiente angular da reta ajustada foi 1,8879; e para a AR, 1,9728. Considerando que o maior valor de $D_{R}$ indica maior rugosidade, 
a dimensão fractal de rugosidade comprovou o resultado da análise morfoscópica, em que a areia de cordão litorâneo $(\mathrm{AJ})$ possui uma textura superficial mais lisa do que a areia de rio (AR).

\section{CONCLUSÕES}

A análise de imagens aliada ao grau de esfericidade e à teoria dos fractais apresentou-se como alternativa viável para obtenção das medidas necessárias para calcular a forma e textura dos grãos de areias.

Os resultados para as duas areias foram analisados estatisticamente para a variável esfericidade, evidenciando diferenças estatísticas significativas entre as duas areias de origens diferentes.

A areia AJ apresentou grau de esfericidade ligeiramente superior e textura superficial mais lisa do que a $A R$, indicando que as areias de jazida (AJ) sofreram maior intemperismo por causa do transporte.

\section{REFERÊNCIAS}

Araújo GS. Estudos de parâmteros texturais das areias para argamassas de revestimento através da análise de imagens [dissertação]. Vitória: Universidade Federal do Espírito Santo; 2001.

Associação Brasileira de Normas Técnicas - ABNT. NBR 248:2003 - Agregados: Determinação da composição granulométrica. Rio de Janeiro: Associação Brasileira de Normas Técnicas 2003 [acesso 2014 Fev 20]. Disponível em: http://www.abntcatalogo.com.br/norma.aspx? ID=2979.

Brzezicki JM, Kasperkiewicz J. Automatic image analysis in evaluation of aggregate shape. J Comp Civil Eng ASCE. 1999;13:123-8.
Bunde A, Havlin S. Fractals in science. Berlin: Springer-Verlag; 1995.

Carr JR, Norris GM, Newcomb DE. Characterization of aggregate shape using fractal dimension. Transp Res Record. 1990;1278:43-50.

Empresa Brasileira de Pesquisa Agropecuária - Embrapa. Padronização de métodos para análise granulométrica no Brasil. Rio de Janeiro; 2012.

Fernlund JMR. Image analysis method for determining 3-D shape of coarse aggregate. Cement Concrete Res. 2005;35:1629-37.

Hyslip JP, Vallejo LE. Fractal analysis of the rougness and size distribution of granular materials. Eng Geol. 1997;48:231-44.

Kwan AKH, Mora CF, Chan HC. Particle shape analysis of coarse aggregate using digital image processing. Cement Concrete Res. 1999;29:1403-10.

Lundqvist JE, Akersson U. Image analysis applied to engineering geology, a literature review. B. Eng Geol Environ. 2001;60:117-22.

Maerz NH. Technical and computational aspects of the measurement of aggregate shape by digital image analysis. J Comp Civil Eng ASCE. 2004;18:10-8.

Mclane M. Sedimentology. New York: Oxford University Press; 1995.

Mendes JC. Estratigrafia e sedimentologia: Geologia estrutural e aerofotogeologia. Brasília: Instituto Nacional do Livro; 1972.

Mora CF, Kwan AKH. Sphericity, shape factor and convexity measurement of coarse aggregate for concrete using digital image processing. Cement Concrete Res. 2000;30:351-8.

Persson AL. Image analysis of shape and size of fine aggregates. Eng Geol. 1998;50:177-86.

Rittenhouse G. A visual method of estimating two-dimensional sphericity. J Sedim Petrol. 1943;13:79-81.

Scarlett B. Materials science and technology: A comprehensive treatment. New York: Weinhein; 1996.

Vepraskas MJ, Cassel DK. Sphericity and roundness of sand in coastal plain soils and relationships with soil physical properties. Soil Sci Soc Am J. 1987;51:1008-112. 